\title{
ESTADO NUTRICIONAL \\ E AS COMORBIDADES ASSOCIADAS \\ AO DIABETES MELLITUS TIPO 2 NO IDOSO
}

Carina Garcia ${ }^{1}$

Maiara de Queiroz Fischer

Fabiana Assmann Poll ${ }^{3}$

resumo

O diabetes mellitus tipo 2 é uma doença metabólica caracterizada por hiperglicemia que ocorre tanto por resistência à ação da insulina como por deficiência na secreção deste hormônio. O objetivo deste estudo foi avaliar e relacionar o estado nutricional, as comorbidades e complicações associadas ao diabetes mellitus tipo 2 em pacientes idosos internados em um hospital do município de Santa Cruz do Sul, RS. A amostra foi composta por 25 pacientes idosos, internados em outubro e novembro de 2014. Foram verificados nos prontuários o gênero, o estado nutricional pelo índice de massa corporal

1 Graduada em Nutrição. Residente no Programa de Residência Multiprofissional em Nutrição do Hospital Santa Cruz. Universidade de Santa Cruz do Sul (UNISC). E-mail: carinagarcia@unisc.br.

2 Graduada em Nutrição. Residente no Programa de Residência Multiprofissional em Nutrição do Hospital Santa Cruz. Universidade de Santa Cruz do Sul (UNISC). E-mail: maiarafischer@unisc.br.

3 Graduada em Nutrição. Mestre em Ciências Médicas Pediatria. Docente do Departamento de Educação Física e Saúde. Universidade de Santa Cruz do Sul (UNISC). E-mail: fpoll@unisc.br. 
(IMC) e a presença de comorbidades e complicações relacionadas ao diabetes. Conforme o IMC, 8\% ( $n=2)$ dos idosos apresentaram magreza, 32\% ( $n=8)$, eutrofia e 60\% ( $n=15)$, excesso de peso. As comorbidades e complicações mais prevalentes foram hipertensão arterial sistêmica (HAS) (84\%), doença arterial coronariana (36\%), trombose (16\%), pé diabético (16\%), amputação de membro inferior (16\%), insuficiência cardíaca congestiva (16\%) e dislipidemia (16\%). Os resultados demonstraram a predominância do excesso de peso, sendo o sexo feminino o mais afetado, e a HAS como a comorbidade mais prevalente entre os pacientes diabéticos.

palavras-chave

Estado Nutricional. Complicações do Diabetes. Diabetes Mellitus Tipo 2.

\section{Introdução}

O diabetes mellitus (DM) é uma doença crônica não transmissível de alta prevalência e incidência no Brasil e no mundo, e por isso tornou-se um grave problema de saúde pública (FUSCALDI; SARTORE; GROSSI, 2011). Estima-se que aproximadamente 150 milhões de pessoas no mundo são portadoras do diabetes mellitus tipo 2 (DM2), com projeção de alcançar 300 milhões em 2025 (LAFERRÈRE et al., 2008). No Brasil, a prevalência de DM na população de 30 a 69 anos é de 7,6\%, o que representa cerca de 10 milhões de pessoas (MALERBI; FRANCO, 1992).

O DM2 é uma doença metabólica de etiologia múltipla e caracterizada por hiperglicemia que ocorre tanto por resistência à ação da insulina como por deficiência na secreção deste hormônio (SBNPE; ABRAN, 2011). Além disso, ocorre distúrbio no metabolismo dos carboidratos, lipídios e proteínas (MARINHO et al., 2012).

Em longo prazo, a hiperglicemia provoca alterações e complicações crônicas, classificadas como: microangiopáticas - a retinopatia diabética, que pode levar à perda de visão, e a nefropatia diabética, que pode evoluir até a falência renal; neuropáticas - a neuropatia periférica, que aumenta o risco de úlcera no pé, podendo evoluir para amputação; e macroangiopáticas - a doença arterial coronariana, doença cerebrovascular e vascular periférica (FERREIRA et al., 2013). A duração da doença e o seu controle interagem com outros fatores de risco, denominados comorbidades, como hipertensão 
arterial sistêmica (HAS), dislipidemia e obesidade, que determinam o curso da microangiopatia e da macroangiopatia (FERREIRA et al., 2013).

Conforme a American Diabetes Association (ADA, 2013), o DM2 é a forma mais comum da doença, sendo encontrado em mais de $90 \%$ da população diabética. Geralmente, surge depois dos 40 anos de idade e tem relação com a obesidade (FUSCALDI; SARTORE; GROSSI, 2011; STOLL et al., 2013).

A gordura abdominal pode aumentar o risco de desencadear o DM2 em 10 vezes. Para cada aumento de $10 \%$ no peso corporal, há aumento de $2 \mathrm{mg} / \mathrm{dL}$ na glicemia em jejum (VARASCHIM et al., 2012). Cerca de $90 \%$ dos portadores de DM2 estão com excesso de peso (ZHOU et al., 2009).

O DM2 é causado por fatores genéticos e pelo estilo de vida. Os genes que predispõem um indivíduo a ter diabetes são considerados essenciais para o desenvolvimento da doença, entretanto a ativação de uma predisposição genética exige a presença dos fatores ambientais e comportamentais, principalmente aqueles associados ao estilo de vida. Neste contexto, estão envolvidos fatores de risco modificáveis e não modificáveis na eclosão do DM2 (ALBERTI; ZIMMET; SHAW, 2007). Dentre os fatores de risco modificáveis, estão o excesso de peso, a gordura abdominal, o sedentarismo e a dieta (MARINHO et al., 2012).

As principais complicações responsáveis por morbidade e mortalidade em pacientes com DM2 são as alterações microvasculares e macrovasculares que geram disfunção, dano ou falência de vários órgãos induzindo os diabé ticos a apresentarem elevado risco de doença vascular aterosclerótica, como as doenças coronarianas, arterial periférica e vascular cerebral, que representam a principal causa de morte (52\%). O estudo clínico denominado de Diabetes Control and Complications Trial (DCCT) e o United Kingdom Prospective Diabetes Study (UKPDS) concluíram que o risco dessas complicações é diretamente proporcional à hiperglicemia (SANTOS; BALZANELI; ANDRADE, 2009). Além disso, o UKPDS mostrou que quanto menor o nível de hemoglobina glicada (A1C), menor o risco de complicações (NASCIMENTO et al., 2010).

O tratamento do DM consiste na manutenção do valor de glicemia e de insulina normal. Esse controle é fundamental para a prevenção de complicações agudas e crônicas decorrentes da doença (FUSCALDI; SARTORE; GROSSI, 2011; VARASCHIM et al., 2012). É consenso que a alimentação saudável deve fazer parte do tratamento do DM, bem como o exercício físico e a medicação (VANCEA et al., 2009).

Conforme Torres, Pereira e Alexandre (2011), é fundamental que o indivíduo portador de DM2 adote habilidades de autocuidado que permita o controle da doença. Quanto maior o acesso à informação e ao conhecimento 
sobre sua comorbidade, maior será a sua capacidade de instituir medidas que lhe permitam a adoção de uma alimentação saudável e a prática de atividade física diária, que irá refletir diretamente na melhoria de sua qualidade de vida (TORRES; PEREIRA; ALEXANDRE, 2011).

Motivado por essa necessidade, a identificação do perfil dos pacientes diabéticos, em especial, os idosos internados, no que se refere ao seu estado nutricional e às comorbidades, pode auxiliar na compreensão da condição de saúde dessa população para que repercute na sua capacidade funcional, na carga de cuidados sobre a família e nos custos com serviços de saúde.

Diante do exposto, este estudo teve como objetivo avaliar o estado nutricional, e relacioná-lo com as comorbidades e complicações associadas ao DM2 de pacientes idosos internados num hospital do município de Santa Cruz do Sul, RS.

\section{Metodologia}

Realizou-se um estudo quantitativo, de corte transversal, composto por pacientes idosos internados num hospital do município de Santa Cruz do Sul, RS, no período de outubro a novembro de 2014. Os dados referentes a gênero e idade, assim como os clínicos, alusivos ao diagnóstico, às comorbidades e às complicações da doença foram coletados dos prontuários desse hospital, junto aos registros médicos. Da mesma forma, obtiveram-se as medidas de peso e estatura, que são verificadas pelos nutricionistas e acadêmicos de nutrição nas rotinas de atendimento, que seguem a Norma Técnica do Sistema de Vigilância Alimentar e Nutricional - SISVAN (BRASIL, 2011). Na impossibilidade de obtenção dessas medidas, utilizam-se as fórmulas de estimativa de peso e estatura propostas por Chumlea, Roche e Steinbaugh (1985). Para classificar o estado nutricional, calculou-se o IMC e classificou-se conforme propõe Lipschitz (1994).

Os aspectos éticos foram previstos através da assinatura do Termo de Consentimento Livre e Esclarecido (TCLE) no momento da internação. A apresentação e anuência do TCLE no momento da internação é uma rotina da instituição por se tratar de um hospital de ensino, que autoriza o uso de dados contidos no prontuário dos pacientes respeitando aos critérios éticos, como prevê a resolução do CNS 466/12.

Os dados foram tabulados e avaliados nos programas Microsoft Excel for Windows versão 2010 e Statistical Package for Social Sciences (SPSS) versão 20.0. 
As variáveis foram analisadas utilizando-se os testes de frequência descritiva e qui quadrado, e o nível de significância utilizado foi de $\mathrm{p}<0,05$.

\section{Resultados}

Participaram do estudo 25 idosos com o diagnóstico de DM2, com idade média \pm DP de $67 \pm 6,8$ anos. Destes, $56 \%$ ( $n=14$ ) eram do sexo feminino. Em relação ao IMC, $8 \%(n=2)$ dos indivíduos apresentaram magreza, $32 \%(n=8)$, eutrofia e $60 \%$ ( $n=15)$, excesso de peso, sendo que destes últimos, 78,6\% ( $n=11)$ eram mulheres (Tabela 1). No entanto, não houve significância estatística em relação ao sexo $(\mathrm{p}=0,064)$.

Tabela 1 - Estado nutricional ${ }^{\star}$ dos pacientes com DM2 internados no hospital no período avaliado.

\begin{tabular}{l|c|c}
\hline \multicolumn{1}{c|}{ IMC } & SEXO FEMININO N (\%) & SEXO MASCULINO N (\%) \\
\hline Magreza & $0(0)$ & $2(18,2)$ \\
\hline Eutrofia & $3(21,4)$ & $5(45,4)$ \\
\hline Excesso de peso & $11(78,6)$ & $4(36,4)$ \\
\hline Total & $14(100)$ & $11(100)$ \\
\hline
\end{tabular}

*Segundo a classificação do IMC para idoso (LIPSCHTZ, 1994).

Fonte: Tabela elaborada pelas autoras

As comorbidades e as complicações agudas e crônicas mais prevalentes nos pacientes diabéticos foram HAS e doença arterial coronariana (Tabela 2). Foram feitas associações estatísticas entre o estado nutricional e cada uma das comorbidades, porém os valores encontrados não foram significativos para a pesquisa: HAS $(\mathrm{p}=0,729)$; doença arterial coronariana $(\mathrm{p}=0,396)$; pé diabético $(\mathrm{p}=0,204)$; amputação de membro inferior $(\mathrm{p}=0,204)$; dislipidemia $(\mathrm{p}=0,729)$; insuficiência cardíaca congestiva $(\mathrm{p}=0,204)$; trombose $(\mathrm{p}=0,729)$; indicação de amputação $(\mathrm{p}=0,146)$; insuficiência renal $(\mathrm{p}=0,861)$; cardiopatia $(\mathrm{p}=0,376)$; infarto agudo do miocárdio $(\mathrm{p}=0,806)$; embolia $(\mathrm{p}=0,806)$; gangrena úmida $(\mathrm{p}=0,331)$; retinopatia diabética $(\mathrm{p}=0,331)$ e acidente vascular encefálico $(\mathrm{p}=0,707)$. Todos os indivíduos do estudo apresentaram no mínimo uma comorbidade associada ao DM2. 
Tabela 2 - Comorbidades e complicações apresentadas pelos pacientes internados, relacionados ao DM2.

\begin{tabular}{|c|c|c|}
\hline CARACTERÍSTICAS & $\mathrm{N}^{\circ}$ & $\%$ \\
\hline \multicolumn{3}{|c|}{ Hipertensão arterial sistêmica (HAS) } \\
\hline Sim & 21 & 84 \\
\hline Não & 4 & 16 \\
\hline \multicolumn{3}{|c|}{ Doença arterial coronariana } \\
\hline Sim & 9 & 36 \\
\hline Não & 16 & 74 \\
\hline \multicolumn{3}{|l|}{ Pé diabético } \\
\hline Sim & 4 & 16 \\
\hline Não & 21 & 84 \\
\hline \multicolumn{3}{|c|}{ Amputação de membro inferior } \\
\hline Sim & 4 & 16 \\
\hline Não & 21 & 84 \\
\hline \multicolumn{3}{|l|}{ Dislipidemia } \\
\hline $\operatorname{Sim}$ & 4 & 16 \\
\hline Não & 21 & 84 \\
\hline \multicolumn{3}{|c|}{ Insuficiência cardíaca congestiva } \\
\hline Sim & 4 & 16 \\
\hline Não & 21 & 84 \\
\hline \multicolumn{3}{|l|}{ Trombose } \\
\hline Sim & 4 & 16 \\
\hline Não & 21 & 84 \\
\hline \multicolumn{3}{|l|}{ Indicação de amputação } \\
\hline Sim & 3 & 12 \\
\hline Não & 22 & 88 \\
\hline \multicolumn{3}{|l|}{ Insuficiência renal } \\
\hline Sim & 3 & 12 \\
\hline Não & 22 & 88 \\
\hline \multicolumn{3}{|l|}{ Cardiopatia } \\
\hline $\operatorname{Sim}$ & 3 & 12 \\
\hline Não & 22 & 88 \\
\hline \multicolumn{3}{|l|}{ Infarto agudo do miocárdio } \\
\hline Sim & 2 & 8 \\
\hline Não & 23 & 92 \\
\hline
\end{tabular}




\begin{tabular}{c|c|c}
\hline Embolia & 2 & 8 \\
\hline Sim & 23 & 92 \\
\hline Não & & \\
\hline Gangrena úmida & 1 & 4 \\
\hline Sim & 24 & 4 \\
\hline Não & 1 & 96 \\
\hline Retinopatia diabética & 24 & 4 \\
\hline Sim & & 96 \\
\hline Não & 1 & \\
\hline Acidente vascular encefálico & 24 & \\
\hline Sim & & \\
\hline Não & & \\
\hline
\end{tabular}

Fonte: Tabela elaborada pelas autoras

\section{Discussão}

O DM é uma doença bastante limitante, capaz de comprometer a capacidade de realizar atividades diárias, a qualidade de vida e a autonomia do idoso, acarretando complicações encefálicas e cardiovasculares, doença renal, amputações, retinopatia, entre outras. Além disso, é uma das principais causas de morte precoce devido ao aumento do risco para as doenças cardiovasculares, as quais contribuem em cerca de $50 \%$ a $80 \%$ para as mortes dos diabéticos (FRANCISCO et al., 2010).

Mudanças no estilo de vida caracterizado por aumento da ingestão calórica e redução da atividade física promoveram juntamente com o sobrepeso e a obesidade o aumento da prevalência do DM, uma vez que são fatores de risco para a doença (ADA, 2008). Frequentemente, o DM acomete mais as mulheres que já tiveram diabetes gestacional, e indivíduos hipertensos ou dislipidêmicos, podendo variar de acordo com a raça e a etnia (ADA, 2013).

Nesta pesquisa, $56 \%$ dos indivíduos eram do sexo feminino e com predominância de excesso de peso, assim como no estudo de Aurichio, Rebelatto e Castro (2010), que mostrou maior prevalência de DM no sexo feminino, sugerindo que há relação entre diabetes e obesidade.

$\mathrm{Na}$ amostra estudada, $60 \%$ dos diabéticos estavam com excesso de peso. Entre as mulheres, $78,6 \%$ estavam classificadas com excesso de peso, enquanto que nos homens, $36,4 \%$, ou seja, os resultados mostram maior prevalência de obesidade em mulheres idosas quando comparadas aos homens da mesma faixa etária. A obesidade é um fator de risco significativo para o aumento da incidência de DM2 e da morbi-mortalidade por outras doenças como dislipidemia, 
HAS, doença coronariana isquêmica, entre outras (MENEZES et al., 2014). Essa condição nutricional merece atenção, apesar dos achados neste estudo serem estatisticamente não significativos em relação às comorbidades, que estiveram presentes em todos os indivíduos portadores de DM2 internados no período avaliado, sendo que estes, em sua maioria, estavam com excesso de peso.

Scheffel et al. (2004) avaliaram a prevalência das complicações crônicas em pacientes com DM2 e aferiram os seus possíveis fatores de risco no Sul do Brasil, sendo que esse estudo demonstrou que $64 \%$ dos indivíduos diabéticos estavam com o colesterol total elevado, 36\% estavam com o IMC $>30 \mathrm{~kg} / \mathrm{m}^{2} \mathrm{e}$ cerca de $50 \%$ estavam com os triglicerídeos alterados.

O estudo populacional realizado em Florianópolis, SC, mostrou que o excesso de peso foi positivamente associado ao DM independentemente de sexo, idade, renda mensal, escolaridade, atividade física regular e HAS (PELEGRINI et al., 2011).

Sabe-se que a HAS é considerada um dos principais fatores de risco para a instalação e a progressão das complicações crônicas do DM2 (VANCEA et al., 2009). Da mesma forma, o seu controle resulta na diminuição de dano aos órgãos-alvo (MALFATTI; ASSUNÇÃO, 2011) e, no presente estudo, foi a comorbidade associada que teve a maior prevalência, semelhante ao estudo de Araújo et al. (2013), que relata um índice 72,9\%.

Nos Estados Unidos, aproximadamente 20\% dos indivíduos com 65 anos ou mais são acometidos por DM2. Além disso, essas pessoas têm outras doenças associadas como a HAS e a obesidade. O DM2 é uma doença preocupante em virtude, sobretudo, dos riscos de desenvolvimento de complicações microvasculares e macrovasculares, que reduzem a expectativa e a qualidade de vida dos pacientes (ARAÚJO et al., 2013).

No estudo, os idosos apresentaram também HAS (84\%) e dislipidemia (16\%), o que aumenta fortemente o risco da doença aterosclerótica. A manutenção do nível ideal de lipídios constitui um dos objetivos do tratamento dos pacientes diabéticos, justamente para evitar a progressão das complicações macrovasculares secundárias ao diabetes (SANTOS; BALZANELI; D'ANDRADE, 2009).

Nossos achados demonstraram que 36\% dos idosos diabéticos apresentavam doença arterial coronariana. Outros estudos relacionam que a gravidade da doença aterosclerótica é significativamente maior em pacientes diabéticos, correlacionando a glicemia de jejum à gravidade da doença, assim como há uma maior prevalência da doença coronariana, insuficiência cardíaca, acidente vascular cerebral e insuficiência vascular periférica nesses pacientes em relação aos sem DM, especialmente em mulheres (FANTIN et al., 2012; SPOSITO et al., 2007). 
Dezesseis por cento dos diabéticos internados apresentavam o pé diabético e $16 \%$ já tinham sofrido amputação de membro inferior. O pé diabético é uma das mais devastadoras complicações crônicas do DM devido ao significativo número de casos que evoluem para amputação. A lesão, que ocorre nos pés dos diabéticos, é decorrente da combinação da neuropatia sensitivo-motora com a autonômica periférica crônica, da doença vascular periférica, das alterações biomecânicas que causam a pressão plantar anormal e da infecção que pode estar presente e agravar ainda mais o caso. O portador de DM tem o risco de amputação 15 vezes maior quando comparado com não diabéticos, correspondendo a 50\% das amputações não traumáticas (COELHO; SILVA; PADILHA, 2009).

Os fatores associados ao desenvolvimento do DM podem ser classificados em três grupos: hereditários, comportamentais e socioeconômicos. Dentre esses, se destacam os fatores de risco comportamentais como alimentação inadequada com a ingestão elevada de alimentos fonte de gorduras trans e saturada, sódio e açúcar; obesidade; sedentarismo; tabagismo e consumo abusivo de bebidas alcoólicas (WHO, 2001). Há evidências científicas de que esses fatores causam a maioria dos novos casos de DM e aumentam o risco de complicações em pessoas diabéticas (MENEZES et al., 2014).

\section{Considerações finais}

Os resultados demonstraram a predominância do excesso de peso nos diabéticos analisados, sendo o sexo feminino o mais afetado. Além disso, deve-se dar atenção especial à HAS, uma vez que foi a comorbidade mais prevalente entre os pacientes diabéticos neste e em outros estudos.

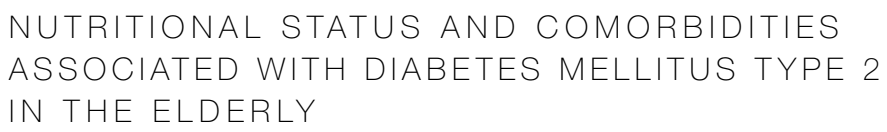

\section{abstract}

Diabetes mellitus type 2 is a metabolic disease characterized by hyperglycemia which occurs both for resistance to insulin as deficiency in the hormone secretion. The objective of this study was to evaluate and relate the nutritional state, the comorbidities and complications associated with the diabetes mellitus type 2 in elderly patients admitted to a hospital in Santa Cruz do Sul, RS, Brazil. 
The sample consisted of 25 elderly patients, admitted in October and November 2014. It was checked in the medical records the gender, the nutritional state by a body mass index (BMI) and the presence of comorbidities and complications associated with the diabetes. According to the BMI, 8\% ( $n=2)$ elderly people showed underweight, $32 \%(n=8)$ eutrophy and 60\% ( $n=15)$ overweight. The most prevalent comorbidities and complications were systemic arterial hypertension $(\mathrm{SAH})(84 \%)$, coronary heart disease (36\%), thrombosis (16\%), diabetic foot (16\%), lower-limb amputation (16\%), congestive heart failure (16\%), and dyslipidemia (16\%). The results showed the predominance of overweight, the female sex being the most affected, and SAH as the most prevalent comorbidity among diabetic patients.

keywords

Nutritional State. Diabetes Complications. Diabetes Mellitus Type 2.

\section{referências}

AMERICAN DIABETES ASSOCIATION. Diagnosis and Classification of Diabetes Mellitus. Diabetes Care, v. 27, n.1, p. 5-10, Jan. 2004. Disponível em: <http://care.diabetesjournals.org/content/27/suppl_1/s5.full>. Acesso em: 10 dez. 2014.

Nutrition Recommendations and Interventions for Diabetes. A position statement of the American Diabetes Association. Diabetes Care, Alexandria, VA, v. 31, Supplement 1, p. S61-S78, Jan. 2008. Disponível em: < http://care.diabetesjournals. org/content/31/Supplement_1/S61.full>. Acesso em: 3 jan. 2015.

ALBERTI, Kurt George Matthew Mayer; ZIMMET, Paul; SHAW, John. International Diabetes Federation: a consensus on Type 2 diabetes prevention. Diabetic Medicine, Oxford, v. 24, n. 5, p. 451-463, May 2007.

ARAÚJO, Márcio Flávio Moura et al. Uso de medicamentos, glicemia capilar e índice de massa corpórea em pacientes com diabetes mellitus. Revista Brasileira de Enfermagem, Brasília, v. 66, n. 5, p. 709-714, set./out. 2013.

AURICHIO, Thaís Rabiatti; REBELATTO, José Rubens; CASTRO, Alessandra Paiva de. Obesidade em idosos do Município de São Carlos, SP e sua associação com diabetes melito e dor articular. Fisioterapia e Pesquisa, São Paulo, v. 17, n. 2, p. 114-117, abr./jun. 2010.

BRASIL. Ministério da Saúde. Secretaria de Atenção à Saúde. Departamento de Atenção Básica. Orientações para a coleta e análise de dados antropométricos em serviços de saúde: Norma Técnica do Sistema de Vigilância Alimentar e Nutricional - SISVAN. Brasília: Ministério da Saúde, 2011. 76 p. Série G. Estatística e Informação em Saúde.

CHUMLEA, William Cameron; ROCHE, Alex F.; STEINBAUGH, Maria L. Estimating stature from knee height for persons 60 to 90 years of age. Journal of the American Geriatrics Society, New York, v. 33, n. 2, p. 116-120, Feb. 1985 
COELHO, Maria Seloi; SILVA, Denise Maria Guerreiro Vieira da; PADILHA, Maria Itayra de Souza. Representações sociais do pé diabético para pessoas com diabetes mellitus tipo 2. Revista da Escola de Enfermagem da Universidade de São Paulo, São Paulo, v. 43, n. 1, p. 65-71, mar. 2009.

FANTIN, Simone de Souza et al. Associação entre diabetes mellitus e gravidade da doença arterial coronariana em pacientes submetidos à intervenção coronária percutânea. Revista Hospital de Clínicas de Porto Alegre, Porto Alegre, v. 32, n. 3, p. 291-295, 2012.

FERREIRA, Juliana Mota et al. Alterações auditivas associadas a complicações e comorbidades no diabetes mellitus tipo 2. Audiology Communication Research, São Paulo, v. 18, n. 4, p. 250-259, dez. 2013.

FRANCISCO, Priscila Maria Stolses Bergamo et al. Diabetes auto-referido em idosos: prevalência, fatores associados e práticas de controle. Cadernos de Saúde Pública, Rio de Janeiro, v. 26, n. 1, p. 175-184, jan. 2010.

FUSCALDI, Fernanda Silva; BALSANELLI, Alessandra Cristina Sartore; GROSSI, Sonia Aurora Alves. Lócus de controle em saúde e autoestima em portadores de diabetes mellitus tipo 2. Revista da Escola de Enfermagem da USP, São Paulo, v. 45, n. 4 p. 855-861, ago. 2011

LAFERRĖRE, Blandine et al. Effect of weight loss by gastric bypass surgery versus hypocaloric diet on glucose and incretin levels in patients with type 2 diabetes. The Journal of Clinical Endocrinology and Metabolism, Chevy Chase, MD, v. 93, n. 7 , p. 2479-2485, July 2008.

LIPSCHTZ, David A. Screening for nutritional status in the elderly. Prim Care, Philadelphia, PA, v. 21, n. 1, p. 55-67, Mar. 1994.

MALERBI, Domingos A.; FRANCO, Laercio J. Multicenter study of the prevalence of diabetes mellitus and impaired glucose tolerance in the urban Brazilian population age 30-69 years. Diabetes Care, Alexandria, VA, v. 15, n. 11, p. 1509-.516, Nov. 1992. Disponível em: <http://care.diabetesjournals.org/content/15/11/1509.abstract> Acesso em: 19 dez. 2014.

MALFATTI, Carlos Ricardo Maneck; ASSUNÇÃO, Ari Nunes. Hipertensão arterial e diabetes na Estratégia de Saúde da Família: uma análise da frequência de acompanhamento pelas equipes de Saúde da Família. Ciência \& Saúde Coletiva, Rio de Janeiro, v. 16, supl. 1, p. 1383-1388, 2011.

MARINHO, Niciane Bandeira Pessoa et al. Diabetes mellitus: fatores associados entre usuários da Estratégia Saúde da Família. Acta Paulista de Enfermagem, São Paulo, v. 25, n. 4, p. 595-600, jul. 2012.

MENEZES, Tarciana Nobre et al. Diabetes mellitus referido e fatores associados em idosos residentes em Campina Grande, Paraíba. Revista Brasileira de Geriatria e Gerontologia, Rio de Janeiro, v. 17, n. 4, p. 829-839, out./dez. 2014.

NASCIMENTO, Alexandra Bulgarelli et al. A relação entre polifarmácia, complicações crônicas e depressão em portadores de Diabetes Mellitus Tipo 2. Revista da Escola de Enfermagem da Universidade de São Paulo, São Paulo, v. 44, n. 1, p. 40-46, mar. 2010.

PELEGRINI, Andreia et al. Diabetes mellitus auto-referido e sua associação com excesso de peso em idosos. Revista Brasileira de Cineantropometria \& Desempenho Humano, Florianópolis, v. 13, n. 6, p. 442-447, nov./dez. 2011

SANTOS, Fernanda Bernardes Fernandes; BALZANELI, Estela Silva; D'ANDRADE, Márcia Regina Pessoa. Avaliação do perfil lipídico de pacientes diabéticos e hipertensos tratados com captopril. Jornal Brasileiro de Patologia e Medicina Laboratorial, Rio de Janeiro, v. 45, n. 3, p. 207-212, jun. 2009. 
SCHEFFEL, Rafael Selbach et al. Prevalência de complicações micro e macrovasculares e de seus fatores de risco em pacientes com diabetes melito do tipo $2 \mathrm{em}$ atendimento ambulatorial. Revista da Associação Médica Brasileira, São Paulo, v. 50, n. 3, p. 263-267, jul./set. 2004

SOCIEDADE BRASILEIRA DE NUTRIÇÃO PARENTERAL E ENTERAL; ASSOCIAÇÃO BRASILEIRA DE NUTROLOGIA. Terapia Nutricional no Diabetes Mellitus. Projeto Diretrizes, v. 9, 8 jul. 2011. Disponível em: <http://www.projetodiretrizes.org.br/9_volume/ terapia_nutricional_no_diabetes_mellitus.pdf>. Acesso em: 18 dez. 2014.

SPOSITO, Andrei et al (Coord.). IV Diretriz Brasileira sobre dislipidemias e prevenção da aterosclerose: Departamento de Aterosclerose da Sociedade Brasileira de Cardiologia. Arquivos Brasileiros de Cardiologia, São Paulo, v. 88, supl. 1, p. 2-19, abr. 2007.

STOLL, Aluisio et al. O efeito em curto prazo do bypass gástrico sobre pacientes obesos diabéticos. Revista do Colégio Brasileiro de Cirurgiões, Rio de Janeiro, v. 40, n. 1, p. 11-15, jan./fev. 2013.

TORRES, Heloísa de Carvalho; PEREIRA, Flávia Rodrigues Lobo; ALEXANDRE, Luciana Rodrigues. Avaliação das ações educativas na promoção do autogerenciamento dos cuidados em diabetes mellitus tipo 2. Revista da Escola de enfermagem da USP, São Paulo, v. 45, n. 5, p. 1077-1082, out. 2011

VANCEA, Denise Maria Martins et al. Efeito da freqüência do exercício físico no controle glicêmico e composição corporal de diabéticos tipo 2. Arquivos Brasileiros de Cardiologia, São Paulo, v. 92, n. 1, p. 23-30, jan. 2009.

VARASCHIM, Michelle et al. Alterações dos parâmetros clínicos e laboratoriais em pacientes obesos com diabetes melito tipo 2 submetidos à derivação gastrojejunal em y de Roux sem anel. Revista do Colégio Brasileiro de Cirurgiões, Rio de Janeiro, v. 39, n. 3, p. 178-182, maio/jun. 2012.

WORLD HEALTH ORGANIZATION. Surveillance of risk factors for noncommunicable diseases: the WHO step wise approach. Geneva: WHO, 2001. Disponivel em: <http:// www.who.int/ncd_surveillance/media/en/269.pdf>. Acesso em: 4 dez. 2014.

$\mathrm{ZHOU}$, Liang et al. Prevalence, incidence and risk factors of chronic heart failure in the type 2 diabetic population: systematic review. Current Diabetes Reviews, San Francisco, v. 5, n. 3, p. 171-184, Aug. 2009.

Recebido: 02/10/2015

Aceite Final: 22/08/2016 